\title{
Generalized quantum entropies
}

\author{
A.P. Santos ${ }^{a}$, R. Silva ${ }^{a, b, *}$, J.S. Alcaniz ${ }^{c}$, D.H.A.L. Anselmo ${ }^{a}$ \\ a Universidade Federal do Rio Grande do Norte, Departamento de Física, Natal, RN 59072-970, Brazil \\ b Universidade do Estado Rio Grande do Norte, Departamento de Física, Mossoró, RN 59610-210, Brazil \\ c Observatório Nacional, Rio de Janeiro, RJ 20921-400, Brazil
}

\section{A R T I C L E I N F O}

\section{Article history:}

Received 15 March 2011

Received in revised form 30 June 2011

Accepted 1 July 2011

Available online 8 July 2011

Communicated by C.R. Doering

\section{Keywords:}

Qantum statistical mechanics

Nonextensivity

\begin{abstract}
A B S T R A C T
A deduction of generalized quantum entropies within the Tsallis and Kaniadakis frameworks is derived using a generalization of the ordinary multinomial coefficient. This generalization is based on the respective deformed multiplication and division. We show that the two above entropies are consistent with ones arbitrarily assumed at other contexts.
\end{abstract}

(C) 2011 Published by Elsevier B.V.

\section{Introduction}

The notion of entropy is directly connected to information associated with the degrees of freedom of physical system [1,2]. Recently, a considerable effort has been made toward the development of a mathematical generalization of this concept aiming at better understanding a number of physical systems [3-5]. Some generalizations are the so-called non-Gaussian (nG) statistics which are based on the entropic measure $[3,4]$

$S_{q}=-\sum_{i=1}^{w} p_{i}^{q} \ln _{q}\left(p_{i}\right) \quad$ and $\quad S_{\kappa}=-\sum_{i=1}^{w} p_{i} \ln _{\kappa}\left(p_{i}\right)$,

where $p_{i}$ is the microstate probability, $w$ stands for the number of states and the nG parameters $(q, \kappa)$ take the values $q=1$ and $\kappa=0$ for Gaussian and $q \neq 1$ and $\kappa \neq 0$ for nG statistics. From the mathematical point of view, the above statistics are based on the deformed functions given, respectively, by

$$
\begin{aligned}
& \exp _{q}(p)=[1+(1-q) p]^{1 / 1-q}, \quad \ln _{q}(p)=\frac{p^{1-q}-1}{1-q} \\
& \exp _{\kappa}(p)=\left[\sqrt{1+\kappa^{2} p^{2}}+\kappa p\right]^{1 / \kappa}, \quad \ln _{\kappa}(p)=\frac{p^{\kappa}-p^{-\kappa}}{2 \kappa} .
\end{aligned}
$$

\footnotetext{
* Corresponding author at: Universidade Federal do Rio Grande do Norte, Departamento de Física, Natal, RN 59072-970, Brazil. Tel.: +55 843215 3793x216; fax: +55843215 3791 .

E-mail addresses: alysonpaulo@dfte.ufrn.br (A.P. Santos), raimundosilva@dfte.ufrn.br (R. Silva), alcaniz@on.br (J.S. Alcaniz) doryh@dfte.ufrn.br (D.H.A.L. Anselmo).
}

The effect of nonextensivity and nonaditivity has been largely studied in the context of quantum mechanics. In this particular context, the generalized Bose-Einstein and Fermi-Dirac distributions in nonextensive systems have been investigated by the at least three different methods, namely: i) the asymptotic approximation proposed by Tsallis et al. [6], which derived the expression for the canonical partition function valid for $|q-1| / k_{B} T \rightarrow 0$; ii) the factorization approximation considered by Büyükiliç et al. [7] to evaluate the grand-canonical partition function and iii) the exact approach developed by Rajagopal et al. [8] which derived the exact integral representation for the grand-canonical partition function of nonextensive systems. The connection between the Kaniadakis framework and quantum statistics has been investigated using the maximal entropy principle [3,9], as well as through applications using the relativistic nuclear equation of state in the context of the Walecka quantum hadrodynamics theory [10].

Recently, a proof of the nG quantum H-theorem in the context of both Tsallis and Kaniadakis formalisms was derived by considering statistical correlations under a collisional term from the quantum Boltzmann equation [11,12]. In the Tsallis case, the positiveness of time variation of the quantum entropy $S_{q}^{Q}$ combined with a duality transformation discussed in Refs. [13,14] implied that the nonextensive parameter $q$ must lie in the interval $[0,2]$ (the same is not true for the Kaniadakis case). Additionally, the stationary states are described by quantum $q$ - and $\kappa$-power law extensions of the Fermi-Dirac and Bose-Einstein distributions.

However, in dealing with quantum Tsallis and Kaniadakis frameworks, a particular attention must be paid to the generalization of entropy which plays a fundamental role within the domain of $n G$ quantum H-theorem [11,12]. Specifically, the Bose-Einstein 
and Fermi-Dirac distributions are calculated through a very concrete mathematical basis of combinatorial nature. Our goal in this Letter is to show that the Tsallis and Kaniadakis quantum entropies can be deduced within the context of a generalized combinatorial method, similarly to ordinary Gaussian statistics. In particular, we show that the two above entropies are consistent with ones arbitrarily assumed at other contexts $[11,12,15]$.

This Letter is organized as follows. In Section 2, we give a brief description of the main considerations on the combinatorial structure of the standard quantum entropy. A deduction of the Tsallis and Kaniadakis quantum entropies based on the generalized combinatorial structure is made in Sections 3 and 4, respectively. We summarize the main conclusions in Section 5.

\section{Quantum entropy in standard statistics}

Let us start by representing the main considerations of the combinatorial structure of the standard quantum entropy. ${ }^{1}$ The quantum entropy is calculated through mathematical structure, e.g.: first, we describe the energy eigenstates $\mathrm{W}$ of a quantum gas by considering the arrangement of the $g_{\alpha}$ and $n_{\alpha}$ quantities. In the case of a Bose-Einstein and Fermi-Dirac gas, respectively, we have [1]

$\mathrm{W}_{\{+\}}=\prod_{\alpha} \frac{\left(n_{\alpha}+g_{\alpha}-1\right) !}{n_{\alpha} !\left(g_{\alpha}-1\right) !}$

and

$\mathrm{W}_{\{-\}}=\prod_{\alpha} \frac{g_{\alpha} !}{n_{\alpha} !\left(g_{\alpha}-n_{\alpha}\right) !}$.

Second, by applying the logarithms functions in the above quantities and using the Stirling approximation for large $n$

$\ln (n !)=n[\ln (n)-1], \quad n \gg 1$,

we can calculate, by using the functional $S^{Q}=\ln (W)$, the explicit expressions for standard quantum entropy for Bose-Einstein and Fermi-Dirac gas, in the following form

$\mathrm{S}^{Q}=-\sum_{\alpha}\left[n_{\alpha} \ln \left(n_{\alpha}\right)-\left(n_{\alpha} \pm g_{\alpha}\right) \ln \left(n_{\alpha} \pm g_{\alpha}\right) \pm g_{\alpha} \ln \left(g_{\alpha}\right)\right]$

where the upper signs refer to the Bose-Einstein and the lower signs to the Fermi-Dirac gases.

\section{Tsallis quantum entropy}

In order to obtain the generalized quantum entropy in Tsallis framework [12], let us first introduce the so-called generalized multinomial coefficients proposed in [16]. Next, we present the generalized multinomial coefficients for the Bose-Einstein and Fermi-Dirac gas.

\subsection{Multinomial coefficients}

The main mathematical aspects discussed in Ref. [16] are based on the deformed functions $\ln _{\mathscr{R}}(x)$ and the inverse $\exp _{\mathscr{R}}(x)$, where $\mathscr{R}$ is a set of deformations parameters [16-18]. The above functions follow the relations

\footnotetext{
1 We consider a spatially homogeneous gas of $N$ particles (bosons or fermions) enclosed in a volume $V$. We also assume that this gas is appropriately specified by regarding the states of energy for a single particle in the container as divided up into groups of $g_{\alpha}$ neighboring states, and by stating the number of particles $n_{\alpha}$ assigned to each such group $g_{\alpha}[1]$.
}

$\ln \mathscr{R}(x \otimes \mathscr{R} y)=\ln \mathscr{R}(x)+\ln \mathscr{R}(y)$,

$\exp _{\mathscr{R}}(x) \otimes_{\mathscr{R}} \exp _{\mathscr{R}}(y)=\exp _{\mathscr{R}}(x+y)$,

$\ln _{\mathscr{R}}\left(x \oslash_{\mathscr{R}} y\right)=\ln _{\mathscr{R}}(x)-\ln _{\mathscr{R}}(y)$,

$\exp _{\mathscr{R}}(x) \oslash_{\mathscr{R}} \exp _{\mathscr{R}}(y)=\exp _{\mathscr{R}}(x-y)$,

with $x, y>0$. By using the transformation $X \rightarrow \ln \mathscr{R}(X)$, or applying the $\mathscr{R}$-exponential function on the left-side relations above, we obtain

$x \otimes_{\mathscr{R}} y:=\exp _{\mathscr{R}}[\ln \mathscr{R}(x)+\ln \mathscr{R}(y)]$,

$x \oslash_{R} y:=\exp _{\mathscr{R}}[\ln \mathscr{R}(x)-\ln \mathscr{R}(y)]$.

The above expressions tend to the ordinary ones for specific values of $\mathscr{R}\left(\mathscr{R} \rightarrow \mathscr{R}_{0}\right) .^{2}$ By considering Eq. (8), is possible to define the so-called $\mathscr{R}$-product of $x_{i}$ elements as

$$
\begin{aligned}
& \prod_{\alpha}^{N} \otimes_{\mathscr{R}} x_{\alpha}:=x_{1} \otimes_{\mathscr{R}} x_{2} \otimes_{\mathscr{R}} \cdots \otimes_{\mathscr{R}} x_{N}=\exp _{\mathscr{R}}\left(\sum_{\alpha}^{N} \ln _{\mathscr{R}}\left(x_{\alpha}\right)\right) \\
& \quad\left(x_{\alpha}>0\right) .
\end{aligned}
$$

The expression (10) and the property of the logarithmic function $\ln \left(x^{a}\right)=a \ln (x)$ lead to two generalized factorial operators ${ }^{3}$

$x !_{\{\otimes \mathscr{R}\}}:=\prod_{\alpha}^{x} \otimes_{\mathscr{R}} \alpha=\exp _{\mathscr{R}}\left(\sum_{\alpha}^{x} \ln _{\mathscr{R}}(\alpha)\right) \quad(x \in \mathrm{N})$,

and

$x !_{\{\odot \mathscr{R}\}}:=\left[\prod_{\alpha}^{x} \otimes_{\mathscr{R}} \alpha^{-1}\right]^{\otimes_{\mathscr{R}}^{-1}}=\exp _{\mathscr{R}}\left(-\sum_{\alpha}^{x} \ln _{\mathscr{R}}(1 / \alpha)\right)$

$(x \in \mathrm{N})$

The multinomial coefficients, constructed through Eqs. (11) and (12), are the basis from the calculation of the Tsallis, Rényi entropy and nonextensive Gaussian entropy [16].

In the present effort, we introduce the deformed multinomial coefficients for the Bose-Einstein and Fermi-Dirac gases, given by

$$
\begin{aligned}
\mathrm{W}_{\{+, \mathscr{R}\}}= & \prod_{\alpha} \otimes \mathscr{R} \\
& \left\{( n _ { \alpha } + g _ { \alpha } - 1 ) ! _ { \{ \otimes \mathscr { R } \} } \oslash _ { \mathscr { R } } \left[n_{\alpha} !_{\{\otimes \mathscr{R}\}}\right.\right. \\
\left.\left.\mathrm{W}_{\{-, \mathscr{R}\}}\left(g_{\alpha}-1\right) !_{\{\otimes \mathscr{R}\}}\right]\right\}^{\otimes_{\mathscr{R}}^{\mathrm{F}(\mathscr{R})},} & \prod_{\alpha} \otimes_{\mathscr{R}}\left\{g _ { \alpha } ! _ { \{ \otimes \mathscr { R } \} } \oslash _ { \mathscr { R } } \left[n_{\alpha} !_{\{\otimes \mathscr{R}\}}\right.\right. \\
& \left.\left.\otimes_{\mathscr{R}}\left(g_{\alpha}-n_{\alpha}\right) !_{\{\otimes \mathscr{R}\}}\right]\right\}^{\otimes_{\mathscr{R}}^{\mathrm{F}(\mathscr{R})}},
\end{aligned}
$$

where $\mathrm{F}(\mathscr{R})$ satisfies the condition $\lim _{\mathscr{R} \rightarrow \mathscr{R}_{0}} \mathrm{~F}(\mathscr{R})=1$.

\subsection{Bose-Einstein gas}

Let us now construct the Bose-Einstein $q$-multinomial coefficient. In this concern, by using $\mathscr{R} \rightarrow 2-q$ in Eq. (13), we get

$$
\begin{aligned}
\mathrm{W}_{\{+, 2-q\}}= & \prod_{\alpha} \otimes_{2-q}\left\{( n _ { \alpha } + g _ { \alpha } - 1 ) ! _ { \{ \otimes 2 - q \} } \oslash _ { 2 - q } \left[n_{\alpha} !_{\{\otimes 2-q\}}\right.\right. \\
& \left.\left.\otimes_{2-q}\left(g_{\alpha}-1\right) !_{\{\otimes 2-q\}}\right]\right\}^{\otimes_{2-q}^{\mathrm{F}(2-q)}},
\end{aligned}
$$

\footnotetext{
2 In the present Letter, we are considering the deformations parameters $q$ and $\kappa$ associated to Tsallis and Kaniadakis statistics, respectively.

3 We emphasize that both definitions of the factorials, Eqs. (11) and (12), provide the same $q, \kappa$-quantum entropies. Therefore, in this Letter, we use the definition (11). In the case of Kaniadakis statistic, and using the property $\ln _{K}(1 / x)=-\ln _{\kappa}(x)$, the two factorials in Eqs. (11) and (12) coincide.
} 
where we choose $\mathrm{F}(2-q)=q$ with the condition $\lim _{q \rightarrow 1} \mathrm{~F}(1)=1$ by mathematical convenience.

Here, by taking the q-logarithm in the expression of the $q$-number of states $\mathrm{W}_{\{+, 2-q\}}$, we obtain

$$
\begin{aligned}
& \ln _{2-q}\left(\mathrm{~W}_{\{+, 2-q\}}\right) \\
& =\sum_{\alpha} q\left\{\ln _{2-q}\left[\left(n_{\alpha}+g_{\alpha}-1\right) !_{\{\otimes 2-q\}}\right]\right. \\
& \left.\quad-\ln _{q}\left(n_{\alpha} !_{\{\otimes 2-q\}}\right)-\ln _{2-q}\left[\left(g_{\alpha}-1\right) !_{\{\otimes 2-q\}}\right]\right\} .
\end{aligned}
$$

Now, by using the definition of factorial (11) and the approximation for large quantities $n_{\alpha}$ and $g_{\alpha}$, given by

$\lim _{x \rightarrow \infty} \sum_{\alpha=1}^{x}\left(\alpha^{q-1}\right) \approx \int_{0}^{x} \alpha^{q-1} \mathrm{~d} \alpha=\frac{x^{q}}{q}$,

we arrive to the following expression

$$
\begin{aligned}
\ln _{2-q}\left(\mathrm{~W}_{\{+, 2-q\}}\right) & =\sum_{\alpha} q\left\{\left[\frac{\left(n_{\alpha}+g_{\alpha}\right)^{q}-q}{q(q-1)}\right]-\left[\frac{\left(n_{\alpha}\right)^{q}-q}{q(q-1)}\right]-\left[\frac{\left(g_{\alpha}\right)^{q}-q}{q(q-1)}\right]\right\} \\
= & \sum_{\alpha} q\left\{\frac{\left(n_{\alpha}+g_{\alpha}\right)^{q}}{q}\left[\frac{\left(n_{\alpha}+g_{\alpha}\right)^{1-q}-1}{1-q}\right]\right. \\
& \left.-\frac{n_{\alpha}^{q}}{q}\left[\frac{\left(n_{\alpha}^{1-q}-1\right)}{1-q}\right]-\frac{g_{\alpha}^{q}}{q}\left[\frac{\left(g_{\alpha}^{1-q}\right)}{1-q}\right]\right\} \\
= & \sum_{\alpha}\left[\left(n_{\alpha}+g_{\alpha}\right)^{q} \ln _{q}\left(n_{\alpha}+g_{\alpha}\right)-n_{\alpha}^{q} \ln _{q}\left(n_{\alpha}\right)-g_{\alpha}^{q} \ln _{q}\left(g_{\alpha}\right)\right],
\end{aligned}
$$

or

$$
\begin{aligned}
S_{q}^{\mathrm{T}}= & \ln _{2-q}\left(\mathrm{~W}_{\{+, 2-q\}}\right) \\
= & -\sum_{\alpha}\left[n_{\alpha}^{q} \ln _{q}\left(n_{\alpha}\right)-\left(n_{\alpha}+g_{\alpha}\right)^{q} \ln _{q}\left(n_{\alpha}-g_{\alpha}\right)\right. \\
& \left.-g_{\alpha}^{q} \ln _{q}\left(g_{\alpha}\right)\right],
\end{aligned}
$$

which is the nonadditive quantum entropy for Bose-Einstein gas.

\subsection{Fermi-Dirac gas}

Analogously to the previous result, using the substitution $\mathscr{R} \rightarrow$ $2-q$ in Eq. (14), the $q$-multinominal coefficient is defined as follows

$$
\begin{aligned}
\mathrm{W}_{\{-, 2-q\}}= & \prod_{\alpha} \otimes_{2-q}\left\{g _ { \alpha } ! _ { \{ \otimes 2 - q \} } \oslash _ { 2 - q } \left[n_{\alpha} !_{\{\otimes 2-q\}}\right.\right. \\
& \left.\left.\otimes_{2-q}\left(g_{\alpha}-n_{\alpha}\right) !_{\{\otimes 2-q\}}\right]\right\}^{\otimes_{2-q}^{\mathrm{F}(2-q)}}
\end{aligned}
$$

where $\mathrm{F}(2-q)=q$.

By making similar calculations, we obtain:

$$
\begin{aligned}
& \ln _{2-q}\left(\mathrm{~W}_{\{-, 2-q\}}\right) \\
& =\sum_{\alpha} q\left\{\left[\frac{g_{\alpha}^{q}-q}{q(q-1)}\right]-\left[\frac{n_{\alpha}^{q}-q}{q(q-1)}\right]-\left[\frac{n_{\alpha}^{q}-q}{q(q-1)}\right]\right\} \\
& =\sum_{\alpha} q\left\{\frac{g_{\alpha}^{q}}{q}\left[\frac{g_{\alpha}^{1-q}-1}{1-q}\right]-\frac{n_{\alpha}^{q}}{q}\left[\frac{n_{\alpha}^{1-q}-1}{1-q}\right]\right. \\
& \left.\quad-\frac{\left(g_{\alpha}-n_{\alpha}\right)^{q}}{q}\left[\frac{\left(g_{\alpha}-n_{\alpha}\right)^{1-q}-1}{1-q}\right]\right\}
\end{aligned}
$$

$$
=\sum_{\alpha}\left[-\left(g_{\alpha}-n_{\alpha}\right)^{q} \ln _{q}\left(g_{\alpha}-n_{\alpha}\right)-n_{\alpha}^{q} \ln _{q}\left(n_{\alpha}\right)+g_{\alpha}^{q} \ln _{q}\left(g_{\alpha}\right)\right],
$$

or

$$
\begin{aligned}
\mathrm{S}_{q}^{\mathrm{T}} & =\ln _{2-q}\left(\mathrm{~W}_{\{+, 2-q\}}\right) \\
& =-\sum_{\alpha}\left[n_{\alpha}^{q} \ln _{q}\left(n_{\alpha}\right)+\left(g_{\alpha}-n_{\alpha}\right)^{q} \ln _{q}\left(g_{\alpha}-n_{\alpha}\right)-g_{\alpha}^{q} \ln _{q}\left(g_{\alpha}\right)\right],
\end{aligned}
$$

which is the nonadditive quantum entropy for Fermi-Dirac gas.

Finally, the nonadditive quantum entropy $S_{q}^{T}$, given by expressions (19) and (22), can be written in a more general form, i.e.,

$$
\begin{aligned}
S_{q}^{\mathrm{T}} & =\ln _{2-q}\left(\mathrm{~W}_{\{ \pm, 2-q\}}\right) \\
& =-\sum_{\alpha}\left[n_{\alpha}^{q} \ln _{q}\left(n_{\alpha}\right) \mp\left(g_{\alpha} \pm n_{\alpha}\right)^{q} \ln _{q}\left(g_{\alpha} \pm n_{\alpha}\right) \pm g_{\alpha}^{q} \ln _{q}\left(g_{\alpha}\right)\right],
\end{aligned}
$$

where the upper sign refers to bosons and the lower one to fermions. Note that, when we take the limit $q \rightarrow 1$, the above expression reduces to the standard case of Eq. (6). Note also that the nonextensive quantum entropies for Bose-Einstein and Fermi-Dirac gases, arbitrarily introduced in Refs. [12,15], are exactly equivalent to expression (23), which has been obtained through the generalized combinatorial method. In particular, the $q$-quantum entropy (Eq. (23)) is valid for values of the nonextensive parameter $q$ lying in the interval $q \in[1, \infty)$. However, by using the definition of the factorial (14), we must obtain (23) with the parameter $q \in[0,1]$.

\section{Kaniadakis quantum entropy}

The derivation of the generalized quantum entropy in the context of the Kaniadakis statistics is based on the fact that the Kaniadakis entropy is related to Tsallis entropy $S_{q}^{(T)}$ through the following expression [3]

$\mathrm{S}_{\kappa}=\frac{\lambda^{\kappa}}{2(1+\kappa)} \mathrm{S}_{1+\kappa}^{(\mathrm{T})}+\frac{\lambda^{-\kappa}}{2(1-\kappa)} \mathrm{S}_{1-\kappa}^{(\mathrm{T})}$,

where $\lambda$ is a real constant. Therefore, we shall use the sum of the two multinomial coefficients constructed in the Tsallis approach. ${ }^{4}$

\subsection{Bose-Einstein gas}

For the Bose-Einstein gas, we use $\mathscr{R} \rightarrow(1+\kappa)$ in (13)

$$
\begin{aligned}
\mathrm{W}_{\{+, 1+\kappa\}}= & \prod_{\alpha} \otimes_{1+\kappa}\left\{( n _ { \alpha } + g _ { \alpha } - 1 ) ! _ { \{ \otimes 1 + \kappa \} } \oslash _ { 1 + \kappa } \left[n_{\alpha} !_{\{\otimes 1+\kappa\}}\right.\right. \\
& \left.\left.\otimes_{1+\kappa}\left(g_{\alpha}-1\right) !_{\{\otimes 1+\kappa\}}\right]\right\}^{\otimes_{1+\kappa}^{\mathrm{F} \alpha}(1+\kappa)}
\end{aligned}
$$

and $\mathscr{R} \rightarrow(1-\kappa)$ in $(14)$

$$
\begin{aligned}
\mathrm{W}_{\{+, 1-\kappa\}}= & \prod_{\alpha} \otimes_{1-\kappa}\left\{( n _ { \alpha } + g _ { \alpha } - 1 ) ! _ { \{ \otimes 1 - \kappa \} } \oslash _ { 1 - \kappa } \left[n_{\alpha} !_{\{\otimes 1-\kappa\}}\right.\right. \\
& \left.\left.\otimes_{1-\kappa}\left(g_{\alpha}-1\right) !_{\{\otimes 1-\kappa\}}\right]\right\}^{\otimes_{1-\kappa}^{\mathrm{F}_{\alpha}(1-\kappa)}}
\end{aligned}
$$

with

$\mathrm{F}_{\alpha}(1 \pm \kappa)=\frac{(1 \mp \kappa)}{2}\left(g_{\alpha}+n_{\alpha}\right)^{ \pm \kappa}$.

\footnotetext{
4 Although there exist many ways to generalize the standard statistic, we are adopting the generalization in the context of the Tsallis expressions. In particular, one similar calculation has been made in previous section.
} 
Now, by taking the $\ln _{1+\kappa}^{(\mathrm{T})}$ and $\ln _{1-\kappa}^{(\mathrm{T})}$, defined by

$\ln _{1+\kappa}^{(\mathrm{T})}=-\frac{x^{-\kappa}-1}{\kappa}, \quad \ln _{1-\kappa}^{(\mathrm{T})}=\frac{x^{\kappa}-1}{\kappa}$,

in Eqs. (25) and (26), respectively, and considering (17) and (27), we obtain

$$
\begin{aligned}
\ln _{1+\kappa}^{(\mathrm{T})}\left(\mathrm{W}_{\{+, 1+\kappa\}}\right) & \\
= & \sum_{\alpha} \mathrm{F}_{\alpha}(1+\kappa)\left\{\ln _{1+\kappa}^{(\mathrm{T})}\left[\left(n_{\alpha}+g_{\alpha}-1\right) !_{\{\otimes 1+\kappa\}}\right]\right. \\
& \left.\quad-\ln _{1+\kappa}^{(\mathrm{T})}\left(n_{\alpha} !_{\{\otimes 1+\kappa\}}\right)-\ln _{1+\kappa}^{(\mathrm{T})}\left[\left(g_{\alpha}-1\right) !_{\{\otimes 1+\kappa\}}\right]\right\} \\
= & \sum_{\alpha} \mathrm{F}_{\alpha}(1+\kappa)\left[-\frac{\left(g_{\alpha}+n_{\alpha}\right)^{1-\kappa}-(1-\kappa)}{\kappa(1-\kappa)}+\frac{n_{\alpha}^{1-\kappa}-(1-\kappa)}{\kappa(1-\kappa)}\right. \\
& \left.+\frac{g_{\alpha}^{1-\kappa}-(1-\kappa)}{\kappa(1-\kappa)}\right] \\
= & \sum_{\alpha} \frac{1}{2 \kappa}\left[-\left(g_{\alpha}+n_{\alpha}\right)+n_{\alpha}\left(\frac{n_{\alpha}}{g_{\alpha}+n_{\alpha}}\right)^{-\kappa}\right. \\
& \left.+g_{\alpha}\left(\frac{g_{\alpha}}{g_{\alpha}+n_{\alpha}}\right)^{-\kappa}\right]
\end{aligned}
$$

and

$$
\begin{aligned}
\ln _{1-\kappa}^{(\mathrm{T})}\left(\mathrm{W}_{\{+, 1-\kappa\}}\right) & \\
= & \sum_{\alpha} \mathrm{F}_{\alpha}(1-\kappa)\left\{\ln _{1-\kappa}^{(\mathrm{T})}\left[\left(n_{\alpha}+g_{\alpha}-1\right) !_{\{\otimes 1-\kappa\}}\right]\right. \\
& \left.\quad-\ln _{1-\kappa}^{(\mathrm{T})}\left(n_{\alpha} !_{\{\otimes 1-\kappa\}}\right)-\ln _{1-\kappa}^{(\mathrm{T})}\left[\left(g_{\alpha}-1\right) !_{\{\otimes 1-\kappa\}}\right]\right\} \\
= & \sum_{\alpha} \mathrm{F}_{\alpha}(1-\kappa)\left[\frac{\left(g_{\alpha}+n_{\alpha}\right)^{1+\kappa}-(1+\kappa)}{\kappa(1+\kappa)}-\frac{n_{\alpha}^{1+\kappa}-(1+\kappa)}{\kappa(1+\kappa)}\right. \\
& \left.-\frac{g_{\alpha}^{1+\kappa}-(1+\kappa)}{\kappa(1+\kappa)}\right] \\
= & \sum_{\alpha} \frac{1}{2 \kappa}\left[\left(g_{\alpha}+n_{\alpha}\right)-n_{\alpha}\left(\frac{n_{\alpha}}{g_{\alpha}+n_{\alpha}}\right)^{\kappa}-g_{\alpha}\left(\frac{g_{\alpha}}{g_{\alpha}+n_{\alpha}}\right)^{\kappa}\right] .
\end{aligned}
$$

Here, the sum of two expressions (28) and (29) leads to $\kappa$ quantum entropy for the Bose-Einstein gas, given by

$\mathrm{S}_{\kappa}=\ln _{1+\kappa}^{(\mathrm{T})}\left(\mathrm{W}_{\{+, 1+\kappa\}}\right)+\ln _{1-\kappa}^{(\mathrm{T})}\left(\mathrm{W}_{\{+, 1-\kappa\}}\right)$

or

$\mathrm{S}_{\kappa}=-\sum_{\alpha}\left[n_{\alpha} \ln _{\kappa}\left(\frac{n_{\alpha}}{g_{\alpha}+n_{\alpha}}\right)+g_{\alpha} \ln _{\kappa}\left(\frac{g_{\alpha}}{g_{\alpha}+n_{\alpha}}\right)\right]$.

\subsection{Fermi-Dirac gas}

Similarly to the previous results, we have two $\kappa$-multinomial coefficients given by

$$
\begin{aligned}
\mathrm{W}_{\{-, 1+\kappa\}}= & \prod_{\alpha} \otimes_{\kappa}\left\{g _ { \alpha } ! _ { \{ \otimes 1 - \kappa \} } \oslash _ { \kappa } \left[n_{\alpha} !_{\{\otimes 1-\kappa\}}\right.\right. \\
& \left.\left.\otimes_{\kappa}\left(g_{\alpha}-n_{\alpha}\right) !_{\{\otimes 1-\kappa\}}\right]\right\}^{\otimes_{1+\kappa}^{\mathrm{F}}(1+\kappa)}, \\
\mathrm{W}_{\{-, 1-\kappa\}}= & \prod_{\alpha} \otimes_{\kappa}\left\{g _ { \alpha } ! _ { \{ \otimes 1 - \kappa \} } \oslash _ { \kappa } \left[n_{\alpha} !_{\{\otimes 1-\kappa\}}\right.\right. \\
& \left.\left.\otimes_{\kappa}\left(g_{\alpha}-n_{\alpha}\right) !_{\{\otimes 1-\kappa\}}\right]\right\}^{\otimes_{1-\kappa}^{\mathrm{F} \alpha(1-\kappa)}},
\end{aligned}
$$

with $\mathrm{F}_{\alpha}(1 \pm \kappa)$ defined in (27). Again, by taking $\ln _{1+\kappa}^{(\mathrm{T})}$ and $\ln _{1-\kappa}^{(\mathrm{T})}$ in the expressions (32) and (33), using (17) and (27), is possible to show that

$$
\begin{aligned}
\ln _{1+\kappa}^{(\mathrm{T})}\left(\mathrm{W}_{\{-, 1+\kappa\}}\right) & \\
= & \sum_{\alpha} \mathrm{F}_{\alpha}(1+\kappa)\left\{\ln _{1+\kappa}^{(\mathrm{T})}\left(g_{\alpha} !\{\otimes 1+\kappa\}\right)-\ln _{1+\kappa}^{(\mathrm{T})}\left(n_{\alpha} !\{\otimes 1+\kappa\}\right.\right. \\
& \left.\quad-\ln _{1+\kappa}^{(\mathrm{T})}\left[\left(g_{\alpha}-n_{\alpha}\right) !_{\{\otimes 1+\kappa\}}\right]\right\} \\
= & \sum_{\alpha} \mathrm{F}_{\alpha}(1+\kappa)\left[-\frac{g_{\alpha}^{1-\kappa}-(1-\kappa)}{\kappa(1-\kappa)}+\frac{n_{\alpha}^{1-\kappa}-(1-\kappa)}{\kappa(1-\kappa)}\right. \\
& \left.+\frac{\left(g_{\alpha}-n_{\alpha}\right)^{1-\kappa}-(1-\kappa)}{\kappa(1-\kappa)}\right] \\
= & \sum_{\alpha} \frac{1}{2 \kappa}\left[-g_{\alpha}\left(\frac{g_{\alpha}}{g_{\alpha}-n_{\alpha}}\right)^{-\kappa}+n_{\alpha}\left(\frac{n_{\alpha}}{g_{\alpha}-n_{\alpha}}\right)^{-\kappa}\right. \\
& \left.+\left(g_{\alpha}-n_{\alpha}\right)\right]
\end{aligned}
$$

and

$$
\begin{aligned}
\ln _{1-\kappa}^{(\mathrm{T})}\left(\mathrm{W}_{\{-, 1-\kappa\}}\right) & \\
= & \sum_{\alpha} \mathrm{F}_{\alpha}(1-\kappa)\left\{\ln _{1-\kappa}^{(\mathrm{T})}\left(g_{\alpha} !_{\{\otimes 1-\kappa\}}\right)-\ln _{1-\kappa}^{(\mathrm{T})}\left(n_{\alpha} !_{\{\otimes 1-\kappa\}}\right)\right. \\
& \left.\quad-\ln _{1-\kappa}^{(\mathrm{T})}\left[\left(g_{\alpha}-n_{\alpha}\right) !_{\{\otimes 1-\kappa\}}\right]\right\} \\
= & \sum_{\alpha} \mathrm{F}_{\alpha}(1-\kappa)\left[\frac{g_{\alpha}^{\kappa+1}-(\kappa+1)}{\kappa(\kappa+1)}-\frac{n_{\alpha}^{\kappa+1}-(\kappa+1)}{\kappa(\kappa+1)}\right. \\
& \left.-\frac{\left(g_{\alpha}-n_{\alpha}\right)^{\kappa+1}-(\kappa+1)}{\kappa(\kappa+1)}\right] \\
= & \sum_{\alpha} \frac{1}{2 \kappa}\left[g_{\alpha}\left(\frac{g_{\alpha}}{g_{\alpha}-n_{\alpha}}\right)^{\kappa}-n_{\alpha}\left(\frac{n_{\alpha}}{g_{\alpha}-n_{\alpha}}\right)^{\kappa}-\left(g_{\alpha}-n_{\alpha}\right)\right] .
\end{aligned}
$$

By adding Eqs. (34) and (35), we obtain the $\kappa$-quantum entropy for the Fermi-Dirac gas, given by

$\mathrm{S}_{\kappa}=\ln _{1+\kappa}^{(\mathrm{T})}\left(\mathrm{W}_{\{-, 1+\kappa\}}\right)+\ln _{1-\kappa}^{(\mathrm{T})}\left(\mathrm{W}_{\{-, 1-\kappa\}}\right)$

or

$\mathrm{S}_{\kappa}=-\sum_{\alpha}\left[n_{\alpha} \ln _{\kappa}\left(\frac{n_{\alpha}}{g_{\alpha}-n_{\alpha}}\right)-g_{\alpha} \ln _{\kappa}\left(\frac{g_{\alpha}}{g_{\alpha}-n_{\alpha}}\right)\right]$.

Finally, the $\kappa$-quantum entropy $S_{\kappa}$ given by expressions (31) and (37) can be written in a general form, i.e.,

$\mathrm{S}_{\kappa}=-\sum_{\alpha}\left[n_{\alpha} \ln _{\kappa}\left(\frac{n_{\alpha}}{g_{\alpha} \pm n_{\alpha}}\right) \pm g_{\alpha} \ln _{\kappa}\left(\frac{g_{\alpha}}{g_{\alpha} \pm n_{\alpha}}\right)\right]$,

where the upper and lower signs refer to bosons and fermions, respectively. In the limit $\kappa \rightarrow 0$, the above expression reduces to the quantum entropy shown in Eq. (6). Note also that the $\kappa$-quantum entropy assumed in Ref. [11] to derive the quantum H-theorem in Kaniadakis framework is the same expression above which was derived through the generalized combinatorial method.

\section{Final remarks}

In several recent analyses, an expression to the Tsallis and Kaniadakis quantum entropies was arbitrarily assumed [11,12,15]. In this Letter, we have not only shown that these assumptions are 
valid but also have provided a consistent mathematical derivation of $S_{q}^{T}$ and $S_{\kappa}$ using the generalized combinatorial method which is based on the generalization of the factorial [16]. In the Tsallis framework, the calculation follows the so-called $q$-algebra $[17,18]$, and the generalization of multinomial coefficients [16]. In the Kaniadakis derivation, we have considered the entropic relation between Kaniadakis and Tsallis [3] in order to calculate the $\kappa$-quantum entropy through the sum of two $q$-multinomial coefficients.

It should be stressed that for the Tsallis case, although the $q$ quantum entropy is valid in the ranges $q \in[0,1]$ and $q \in[1, \infty)$, using the multinomial coefficients given by Eqs. (11) and (12), respectively, the nonextensive parameter $q$ has been constrained to interval of validity $q \in[0,2]$ through the physical arguments [1214,19], experimental/observational measurement [20] and mathematical considerations [21].

Finally, it should be emphasized that the generalized quantum entropies and the $\mathrm{H}$-theorem associated presents the deformed quantum distributions ( $q, \kappa$-Fermi-Dirac and Bose-Einstein) which are basis for applications at systems in the quantum regime, e.g., nuclear matter [10], Bose-Einstein condensation [22,23] and superconductivity [24].

\section{Acknowledgements}

The authors thank the anonymous referees for their valuable suggestions and comments. R.S. would like to thank the hospitality of the Departamento de Astronomia of Observatório Nacional/MCT where part of this work was developed. A.P.S. acknowledges financial support from CAPES. R.S. and J.S.A. thank CNPq for the grants under which this work was carried out. D.H.A.L.A. acknowledges financial support from Fundação de Amparo à Pesquisa do Estado do Rio Grande do Norte - FAPERN.

\section{References}

[1] R.C. Tolman, The Principles of Statistical Mechanics, Dover, 1979.
[2] J. von Neumann, Mathematische Grundlagen der Quantenmechanik, Springer, Berlin, 1932.

[3] G. Kaniadakis, Physica A 269 (2001) 405;

G. Kaniadakis, Phys. Rev. E 66 (2002) 056125;

G. Kaniadakis, Phys. Rev. E 72 (2005) 036108;

G. Kaniadakis, Europhys. Lett. 92 (2010) 35002.

[4] C. Tsallis, J. Stat. Phys. 52 (1988) 479; M. Gell-Mann, C. Tsallis (Eds.), Nonextensive Entropy: Interdisciplinary Applications, Oxford University Press, New York, 2004.

[5] C. Beck, E.G.D. Cohen, Physica A 322 (2003) 267;

V. Badescu, P.T. Landsberg, J. Phys. A 35 (2002) L591;

V. Badescu, P.T. Landsberg, Complexity 15 (3) (2002) 19.

[6] C. Tsallis, F.C. Sa Barreto, E.D. Loh, Phys. Rev. E 52 (1995) 1447.

[7] F. Büyükiliç, D. Demirhan, A. Gülec, Phys. Lett. A 197 (1995) 209.

[8] A.K. Rajagopal, R.S. Mendes, E.K. Lenzi, Phys. Rev. Lett. 80 (1998) 3907; E.K. Lenzi, R.S. Mendes, A.K. Rajagopal, Phys. Rev. E 59 (1999) 1398.

[9] A. Aliano, G. Kaniadakis, E. Miraldi, Physica B 325 (2003) 35; A.M. Teweldeberhan, H.G. Miller, R. Tegen, Int. J. Mod. Phys. E 12 (2003) 699.

[10] F.I.M. Pereira, R. Silva, J.S. Alcaniz, Phys. Rev. C 76 (2007) 015201;

F.I.M. Pereira, R. Silva, J.S. Alcaniz, Phys. Lett. A 373 (2009) 4214; F.I.M. Pereira, R. Silva, J.S. Alcaniz, Nucl. Phys. A 828 (2009) 136.

[11] A.P. Santos, R. Silva, J.S. Alcaniz, D.H.A.L. Anselmo, Phys. Lett. A 375 (2011) 352.

[12] R. Silva, D.H.A.L. Anselmo, J.S. Alcaniz, Europhys. Lett. 89 (2010) 10004; R. Silva, D.H.A.L. Anselmo, J.S. Alcaniz, Europhys. Lett. 89 (2010) 59902 (Erratum).

[13] J.A.S. Lima, R. Silva, A.R. Plastino, Phys. Rev. Lett. 86 (2001) 2938; R. Silva, A.R. Plastino, J.A.S. Lima, Phys. Lett. A 249 (1998) 401; R. Silva, J.A.S. Lima, Phys. Rev. E 72 (2005) 057101.

[14] I.V. Karlin, M. Grmela, A.N. Gorban, Phys. Rev. E 65 (2002) 036128.

[15] A.M. Teweldeberhan, A.R. Plastino, H.C. Miller, Phys. Lett. A 343 (2005) 71.

[16] Th. Oikonomou, Physica A 386 (2007) 119.

[17] E.P. Borges, Physica A 340 (2004) 95.

[18] L. Nivanen, A. Le Mehaute, Q.A. Wang, Rep. Math. Phys. 52 (2003) 437.

[19] S. Abe, A.K. Rajagopal, Phys. Rev. Lett. 91 (2003) 120601.

[20] S.H. Hansen, D. Egli, L. Hollenstein, C. Salzmann, New Astron. 10 (2005) 379; R. Silva, J.S. Alcaniz, J.A.S. Lima, Physica A 356 (2005) 509; Bin Liu, J. Goree, Phys. Rev. Lett. 100 (2008) 055003;

L.F. Burlaga, N.F. Ness, Astrophys. J. 703 (2009) 311;

J.C. Carvalho, J.D. do Nascimento Jr., R. Silva, J.R. De Medeiros, Astrophys. J. Lett. 696 (2009) L48;

CMS Collaboration, Phys. Rev. Lett. 105 (2010) 022002;

CMS Collaboration, JHEP 1002 (2010) 041.

[21] Th. Oikonomou, G.B. Bagci, Phys. Lett. A 374 (2010) 2225.

[22] B. Tanatar, Phys. Rev. E 65 (2002) 046105.

[23] S. Biswas, Phys. Lett. A 372 (2008) 1574.

[24] Lizardo H.C.M. Nunes, E.V.L. de Mello, Physica A 305 (2002) 340. 